\title{
Incidencia de la lectura crítica para la producción de artículos de opinión
}

Yuidissi Mesa Soledad*

Artículo de reflexión.

Fecha de Recepción: 1 diciembre 2017.

Fecha de Aprobación: 11 abril 2018.

\section{Resumen}

Este artículo es el resultado de la reflexión al proceso investigativo que se llevó a cabo en el trabajo de grado en la Maestría Educación Modalidad Profundización de la UPTC, el cual lleva el mismo nombre de este documento. La propuesta hace énfasis en la puesta en marcha de estrategias que permitan promover la lectura crítica en los estudiantes de grado $8^{\circ}$ para la producción de artículos de opinión. Este estudio se enmarca dentro de un enfoque cualitativo con un diseño de Investigación Acción, utilizando instrumentos de recolección de información como la observación directa y la prueba diagnóstica para la identificación de la problemática; aspecto fundamental para determinar la temática e iniciar la búsqueda de

estrategias metodológicas propuestas por autores como Isabel Solé, Daniel Cassany y Fabio Jurado. El diseño y desarrollo de la propuesta "Critico, luego escribo", logró fortalecer la lectura crítica de los estudiantes y su vez, mejorar las habilidades escriturales, evidenciados en los artículos que fueron publicados en el boletín informativo "Última Edición", medio informativo de la Institución Educativa. Estos textos se caracterizaron por una buena estructura, uso adecuado de la ortografía y dominio de algunas propiedades del texto en relación con la coherencia y cohesión.

Palabras clave: artículos de opinión, lectura crítica, estrategias, estudiante, fortalecer.
* Institución Educativa Vado Castro - Tópoga yuidissi@yahoo.es

* *Universidad Pedagógica y Tecnológica de Colombia Boyacá - Colombia ruby.mendoza@uptc.edu.co

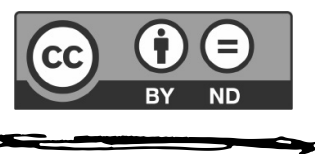




\section{Introducción}

Las actividades de lectura deben desarrollar en los estudiantes habilidades del pensamiento que les ayuden a alcanzar los diferentes niveles de comprensión lectora: literal, inferencial y crítica, esta última conocida también como lectura intertextual. Para tal fin, se hace necesario implementar en las aulas de clase estrategias que conlleven al estudiante a ser un sujeto comprensivo, crítico y reflexivo capaz de analizar la realidad.

Inicialmente, la investigación parte de un proceso de observación que se realizó durante varios meses, ya que el investigador es docente de la población objeto de estudio, donde se identifican dificultades de los estudiantes para asumir en forma crítica; situación que se confirmó con la aplicación de una prueba diagnóstica. En un segundo momento, se promovieron estrategias didácticas tanto para fortalecer la lectura crítica como para elaborar textos de opinión. Por último, se describió el impacto de la propuesta analizando la capacidad argumentativa de los estudiantes en textos informativos a la luz de varios referentes teóricos.

Teniendo en cuenta lo anterior, es importante destacar que la lectura crítica constituye una herramienta de formación del pensamiento crítico, que para autores como Jurado (2014), la definen como:

La lectura crítica no es la "libre opinión” del lector. La lectura crítica surge del ejercicio intelectual que presupone hacer inferencias, sean simples o complejas; las inferencias complejas dependen de las asociaciones entre los conocimientos que promueve el texto (...) es el diálogo entre los conocimientos del texto y los del lector constituye la lectura crítica, que es en sí un juego en el asombro de identificar intencionalidades en lo leído (p.20).

En consecuencia, cuando el proceso de lectura no es satisfactorio se presentan debilidades a la hora de realizar escritos y en el caso de los estudiantes de grado $8^{\circ}$ de la Institución Educativa Vado Castro, la capacidad de producción textual es deficiente en la mayoría de ellos y nula en un pequeño porcentaje. Por consiguiente, los estudiantes no son conscientes que para poder escribir hay que leer y acuden al plagio de información en internet cuando se les asignan actividades escritas o permiten que estas sean realizadas por otras personas. $\mathrm{Al}$ respecto, Argumenta Díaz-Barriga \& Hernández (2012):

La escritura como instrumento cultural ha influido en el desarrollo del pensamiento de la humanidad y puede decirse que también influye en los modos de aproximación de los educandos a su realidad cultural y en su propio desarrollo intelectual (...) En el ámbito académico, leer y escribir pensando son habilidades muy preciadas (p.226).

Es evidente que a través de la producción textual, el estudiante da a conocer sus sentimientos, emociones y puntos de vista en relación con las diferentes situaciones sociales y culturales de 
su entorno, que de una u otra forma, afectan de forma positiva o negativa su vida diaria. En la población donde se realizó la investigación, los jóvenes se ven inmersos en una realidad donde existen notorias dificultades económicas, problemáticas familiares y sociales que afectan el normal desarrollo de la personalidad y por ende, su proyección personal y profesional; aspectos que se pueden identificar en sus escritos.

\section{Referentes teóricos}

En el desarrollo del marco teórico se referencia como categoría principal la lectura crítica, tomando especialmente los aportes de Fabio Jurado Valencia y Daniel Cassany; este último, también es tenido en cuenta en cuanto el proceso de producción textual. De igual manera, se incluyen los parámetros del Ministerio de Educación Nacional en cuanto a los Estándares Básicos de Lenguaje (EBL) y los Derechos Básicos de Aprendizaje (DBA).
Para iniciar, es necesario partir de conceptos básicos como:

¿Qué es leer?

Leer es un ejercicio de comprensión que lleva al conocimiento de la realidad y de igual manera, el desarrollo de competencias en lenguaje para la apropiación del conocimiento, según Solé (1998):

El aprendizaje se construye, registra y comunica mediante el lenguaje $y$, en ese sentido, todos los docentes, independientemente de su área, son docentes de lengua. Cuando un estudiante es capaz de hablar y escribir construye conocimiento, es por eso que una actividad efectiva en el proceso de enseñanza es que se logre la propiedad lingüística y de contenido, para que se evidencie el aprendizaje real y la creación de nuevos conocimientos (p.45).

De esta forma Solé (1998) propone tres etapas importantes que se deben llevar a cabo en la comprensión textual:

Figura 1. Etapas de la comprensión textual

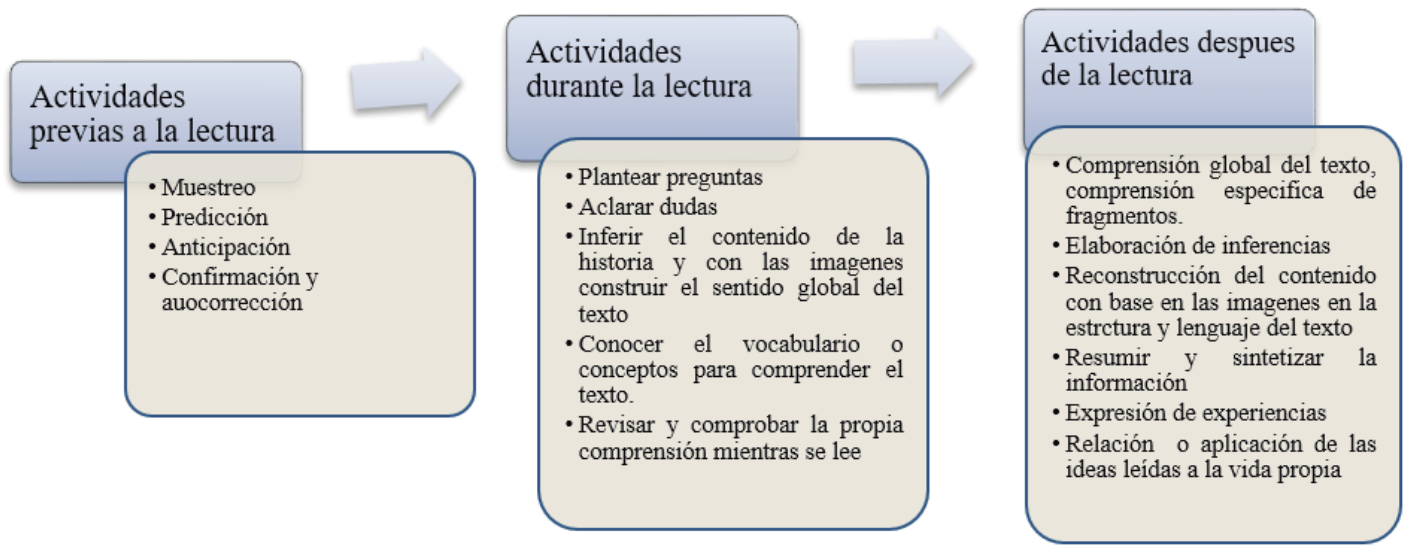

Fuente: Solé (1998) estrategias de lectura. 


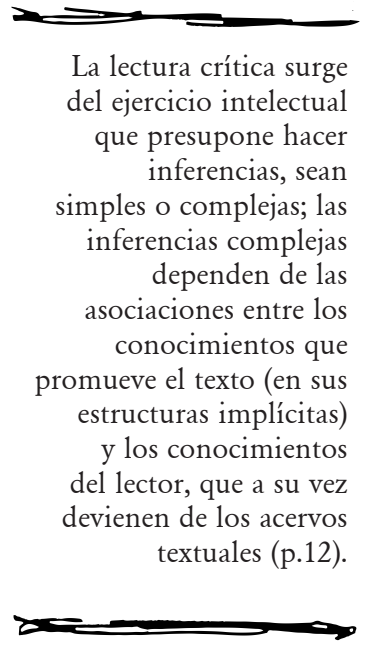

La lectura crítica surge ejercicio intelectual ue presupone hacer mples o complejas; las inferencias complejas dependen de las sociaciones entre los conocimientos que estructuras implícitas) y los conocimientos del lector, que a su vez devienen de los acervos textuales (p.12).
La comprensión lectora está ligada al interés del lector y al contexto, desafortunadamente en la escuela casi siempre se imponen las lecturas que se van a utilizar en las asignaturas y que dejan ver más el interés personal del docente que las necesidades y gustos de los estudiantes. De igual manera, los estudiantes están desarrollando solamente el nivel literal de interpretación textual y en algunos casos se alcanza al inferencial, lo cual no es suficiente para lograr una verdadera competencia comunicativa. En la actualidad se debe enseñar a leer y no solamente en forma comprensiva sino crítica, esto para poder acceder a toda la información que esta era desarrolla, pues los jóvenes se enfrentan a medios de comunicación permanente como internet, redes sociales, y gran cantidad de símbolos y lenguajes no verbales que deben ser abordados en forma adecuada para poder ser asertivos hacia el conocimiento, seleccionando la información más adecuada. Por lo anterior, la lectura es un elemento transversal en el currículo, por cuanto es la herramienta que permite al estudiante acceder a la información que necesita en su proceso de aprendizaje en las diferentes disciplinas y crear nuevos conocimientos; así mismo, le sirve para comprender la realidad del contexto donde se encuentra para proponer alternativas de solución a las dificultades de su entorno y ser parte activa en la construcción de la sociedad.

\section{Lectura crítica}

En relación con la lectura crítica, esta se puede definir a través de dos conceptos: el primero en cuanto a la lectura que hace referencia a decodificar textos y/o es un proceso mental que permite deducir significados de un texto, y el concepto crítica que se define como la opinión, el juicio o evaluación que se hace de un tema determinado, nos argumenta Jurado (2010) citando a Eco (1981):

La lectura crítica surge del ejercicio intelectual que presupone hacer inferencias, sean simples o complejas; las inferencias complejas dependen de las asociaciones entre los conocimientos que promueve el texto (en sus estructuras implícitas) y los conocimientos del lector, que a su vez devienen de los acervos textuales (p.12).

Significa que además de comprender lo que se lee, se realiza un análisis expresando de forma verbal o no verbal lo que expresa en cuanto a aciertos o desaciertos de los textos. Lo anterior tiene estrecha relación con lo que afirma Solé (1998): "Leer es un proceso de interacción entre el lector y el texto, proceso mediante el cual el primero intenta satisfacer (obtener una información pertinente para) los objetivos que guían su lectura” (p. 17).

Por otro lado, la lectura crítica para Cassany (1993), consiste en un "único procedimiento didáctico que fortalece un yo autónomo, consciente y constructivo, con opiniones propias y con capacidades de compromiso con la comunidad" (p.201). Es decir, que el ejercicio de una lectura crítica desarrolla de forma correcta el pensamiento de manera tal que se pueda llevar a cabo con precisión una serie de discursos en 
el ámbito profesional, personal o social, y se asuma una postura propia sobre un tema, considerando las diferentes variables que este pueda tener.

De igual forma, este autor afirma que existen tres planos en la lectura: las -líneas, entre las líneas y detrás de las líneas. Comprender las líneas hace referencia a entender el significado literal; entre las líneas se relaciona con lo que hay que deducir de las palabras, es decir, lo que no está explícitamente: las inferencias, las presuposiciones, la ironía, los dobles sentidos; y lo que hay detrás de las líneas es la ideología, el punto de vista, la intención, la argumentación del autor y se le podría agregar los elementos que aporta el lector desde su contexto situacional, ideológico y cultural; este último hace referencia a la lectura crítica.

En cuanto a los componentes de la lectura crítica, intervienen el lector y el texto, como primera instancia, el texto tiene que ser ojeado y examinado, para identificar aspectos importantes de su estructura, su autor y procedencia, la clase de texto, la intención del autor, el lenguaje utilizado, la temática o tesis del autor y la controversia de su teoría; define Solé (1998):

El lector ante el texto, procesa sus elementos, componentes, empezando por las letras, continuando con las palabras, frases...en un proceso ascendente y secuencial y jerárquico que conduce a la comprensión del texto. Las propuestas de enseñanza que se basan en él atribuyen una gran importancia a las habilidades de descodificación, pues consideran que el lector pue- de comprender el texto porque puede descodificarlo en su totalidad- "modelo top down (p.19).

\section{Competencias y estrategias para la formación del lector crítico}

Las nuevas exigencias del mundo y la diversidad de la lectura en las que se debe acceder a la vida académica hacen necesario que el estudiante incorpore una serie de estrategias para darle significado a un texto, comprenderlo e interpretarlo, en este sentido, parafraseando a los siguientes autores, Girón, Jiménez \& Camargo (2007, p.49123), ellos proponen ejecutar estrategias para formar lectores competentes:

- Valorar la procedencia del texto, el estudiante debe identificar si el texto que va a leer es confiable, es tendencioso, persuasivo y su procedencia.

- Interrogar al texto, consiste en cuestionar el texto, hacerle preguntas, es acá donde el lector descubre si lo que dice el texto es confiable, las preguntas pueden estar encaminadas a situar el texto en el contexto, sociocultural de partida, a identificar la tesis con sus argumentos y a reconocer donde se origina el discurso.

- Relacionar saberes previos, el estudiante compara sus saberes previos con los del texto y aporta desde su conocimiento su punto de vista al contenido textual.

- Contextualizar el texto, el estudiante debe hacer consultas bibliográficas 
en la biblioteca o en el internet del tema que trata el texto, luego compara la información en una ficha de lectura y por último elabora un texto que ponga en relación los textos consultados con el texto original.

- Diferenciar entre bechos y opiniones, el estudiante identifica los hechos y las opiniones en el texto y aporta su punto de vista, respetando el del autor.

- Considerar la opinión del autor, el estudiante identifica la intensión del autor que puede ser: instruir, informar, exponer divertir, aconsejar etc... para esto es indispensable reconocer el tipo de texto, ¿qué motivos tiene el autor para escribir el texto?, ¿con qué fin lo hace?, ¿qué quiere persuadir o informar?

- Determinar controversias, se deben encontrar controversias $\mathrm{O}$ desacuerdos en el texto, en el cual el autor expone puntos de vista de desacuerdo utilizando otras voces que le dan la razón a las opiniones en las que el manifiesta su inconformismo.

- Establecer relaciones de intertextualidad, consiste en identificar las voces de otros textos dentro de un texto con el propósito de dar un alto valor contundente a las razones que da el autor.

- Reconocer analogías, se identifica el uso de la comparación en el texto con el propósito de reafirmar la hipótesis central del texto, esta explica y describe, pero no prueba que lo que describe sea verdadero.
- Asumir una lectura crítica, se deben hacer juicios para evaluar la relevancia y la idoneidad del texto, se debe reflexionar sobre la validez de lo que se ha leído a la luz del conocimiento y de la comprensión del mundo que previamente se tiene.

De conseguirse lo anterior en la formación de los estudiantes, se logrará contar con personas críticas, capaces de asumir con autonomía y responsabilidad su propio pensamiento, de cuestionar y mirar desde diferentes ópticas las situaciones de su entorno; por tal razón, en el aula se requiere fomentar actividades permanentes que permitan el desarrollo de habilidades cognitivas como: el análisis, la crítica, la argumentación a partir de la comprensión textual, la elaboración de textos informativos (como artículos de opinión), que conduzcan a los estudiantes a analizar la problemática sociales culturales, políticas científicas y económicas de un mundo cambiante. Cuando se lee, se elaboran a partir de la información imágenes en nuestra mente, estas poseen significados que el lector utiliza para hacer inferencias y conseguir una representación personal. Lo anterior se sustenta en Díaz-Barriga \& Hernández (2012):

La construcción se elabora a partir de la información que el autor propone en el texto, pero se ve fuertemente enriquecida por las interpretaciones, inferencias, integraciones que el lector adiciona de forma activa, para conseguir así, una representación personal de lo que intentó comunicar de forma explícita o implícita. En este sentido, podemos sostener que 
los significados de un texto son idiosincráticos del lector y cómo hemos dicho no están en el texto "esperando" a ser recuperados por aquel completamente determinados por lo que el autor del texto quiso decir" (p. 228).

\section{Producción textual}

En cuanto a la producción textual, aprender a escribir debe ser un objetivo de la escuela, para Cassany (1993): "escribir es ser capaz de expresar información de forma coherente y correcta para que la entiendan otras personas (...) escribir es una tarea ardua pero no difícil” (p.13) Una de las grandes dificultades que se presentan en el aula es que el docente define lo que se va a escribir, sin que el estudiante exprese su interés. Es por ello que debe haber un cambio y empezar a dar estrategias para que el estudiante empiece a preguntarse ¿A quién escribo?, ¿Para qué escribo?, ¿Sobre qué escribo? y ¿Cómo lo escribo? La producción textual se puede iniciar por la necesidad que crea el ejercicio intelectual, la acción de un interlocutor, la evocación, la lectura, la investigación o cualquier otro estímulo externo o interno, que lleve al individuo a comunicarse de manera oral o escrita, gestual o artística. Por consiguiente, se afirma que la comunicación es una necesidad humana que satisface tanto al que proyecta como al que recibe (Cassany, 1993). De acuerdo con Tobón (2007):

La producción textual va del significado a la expresión fónica (o gráfica), y comienza por la selección de opciones conceptuales, luego, surgen las asociaciones que dan lugar a complejos de estructuras conceptuales que, al formar universos de significado, cumplen principios de restricción semántica, reflejados en relaciones formales entre palabras o grupos de palabras, que, al ser representadas por cadenas fónicas o gráficas, transmiten un sentido al oyente lector. De este modo, todo trabajo de creación de una unidad comunicativa supone tres etapas: Intención, planeación y ejecución ( $\mathrm{p}$. 169).

En relación con las estrategias para la producción textual, Cassany (1993) sustenta las siguientes etapas para el desarrollo del ejercicio escritural:

En la etapa de planificación se debe que tener en cuenta la progresión de actividades hacia la autonomía y hacia textos más complejos. Algunas actividades a realizar en esta etapa pueden ser acostumbrarse a redactar un poco cada día, ya sea tomando notas o escribiendo un diario personal; realizando actividades de preguntas o de representación del pensamiento de manera gráfica (mapas de ideas o mentales, árboles, ideogramas, redes de ideas...); mapas conceptuales; técnicas como el torbellino de ideas, la exploración del tema (con procedimientos como la estrella que deriva de la fórmula periodística de la noticia, en la que ha de especificarse el qué, el quién, el cuándo, el dónde, el cómo y el porqué, o el cubo) y el desenmascaramiento de palabras clave; la escritura libre, frases empezadas, toma de notas. (p.169)

\footnotetext{
La producción textual va del significado a la expresión fónica (o gráfica), y comienza por la selección de opciones conceptuales, luego, surgen las asociaciones que dan lugar a complejos de estructuras conceptuales que, al formar universos de significado, cumplen principios de restricción semántica, reflejados en relaciones formales entre palabras o grupos de palabras, que, al ser representadas por cadenas fónicas o gráficas, transmiten un sentido al oyente lector. De este modo, todo trabajo de creación de una unidad comunicativa supone tres etapas: Intención, planeación y ejecución
} 
La investigación acción educativa no solo se constituye como ciencia práctica y moral, sino también como ciencia crítica, es una forma de indagación autorreflexiva realizada por quienes participan (profesores, alumnados, directivas) en las situaciones sociales (incluyendo las educativas) para mejorar la racionalidad y la justicia de: a) sus propias prácticas sociales o educativas; b) su comprensión sobre las mismas; y c) las situaciones e instituciones en que estas prácticas se realizan (aulas o escuelas)
Por otra parte, en la fase de redacción es ser creativos, por ejemplo, a la hora de escribir documentos más relacionados con la vida que con el colegio se puede empezar rellenando formularios (puesto que la organización de las ideas les viene dada) para pasar a hacer un folleto (Cassany, 1993). Y, por último, la etapa de revisión, una vez haya escrito cada uno su texto, se aplican técnicas auto correctivas. El artículo de opinión se caracteriza por ser un texto periodístico que manifiesta el sentir o el pensar de una persona o medio de comunicación acerca de un asunto que despierta el interés de la opinión pública, "este debe tener las siguientes características; que sea corto, que represente la opinión particular del autor, que contenga temas diversos, un título atractivo y original, subjetividad, estructura y dinámico" (Cassany, 1995, p.201).

\section{Artícullo de opinión}

En relación con el artículo de opinión es un recurso, no sólo para adquirir conocimientos sino también como fuente de lecturas agradables, que ponen en contacto a los niños y jóvenes con temas actuales y de interés. $\mathrm{Al}$ acceder a una lectura por gusto y motivación, el estudiante adquiere información que le permite construir sus propias ideas para manifestar aceptación o rechazo por determinadas situaciones y si se hace en forma escrita, el artículo de opinión es un muy buen recurso, que al ser orientado adecuadamente por los docentes, produce excelentes resultados.

\section{Metodología}

Con el tipo de Investigación Acción (I-A) se busca generar un cambio en el conocimiento educativo, sustenta Kemmis (1984):
La investigación acción educativa no solo se constituye como cien- cia práctica y moral, sino también como ciencia crítica, es una forma de indagación autorreflexiva reali- zada por quienes participan (pro- fesores, alumnados, directivas) en las situaciones sociales (incluyen- do las educativas) para mejorar la racionalidad y la justicia de: a) sus propias prácticas sociales o educa- tivas; b) su comprensión sobre las mismas; y c) las situaciones e ins- tituciones en que estas prácticas se realizan (aulas o escuelas) (p.24).

Se debe entender la I-A como una indagación realizada por el docente investigador para mejorar su práctica pedagógica, a través de la aplicación de estrategias pedagógicas o acciones que ayuden a solucionar deficiencias o problemas en el orden educativo. Para dar respuesta a los objetivos de la investigación se plantean las siguientes fases:

Figura 2. Fases de la investigación.

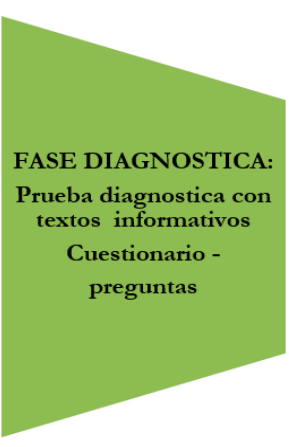

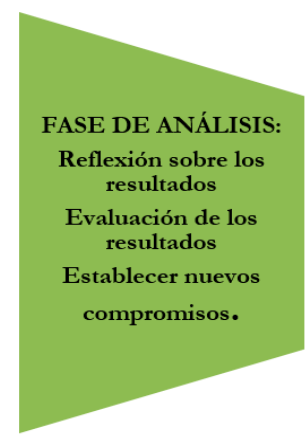

Fuente. Autoría propia. 
La investigación tiene un enfoque cualitativo, los resultados son arrojados de manera descriptiva por su carácter social, el investigador es el principal instrumento de recogida de datos e interacción constante con la realidad objeto de estudio, para Gialdino (2006): "se entiende que entre los rasgos más característicos se encuentra: a). el interés por el significado y la interpretación, b) el énfasis sobre la importancia del contexto y de los procesos y, c) la estrategia inductiva y hermenéutica, considerados como un todo" (p.130) además de esto el tipo de investigación cualitativa es flexible y se va concretando progresivamente. Según Pérez (1994):

La investigación cualitativa se considera como un proceso activo, sistemático, y riguroso de indagación dirigida en el cual se toman decisiones sobre lo investigable en tanto está en el campo de estudio. El foco de atención de los investigadores está en descripciones detalladas de situaciones, eventos, personas, intersecciones y comportamientos que son observables incorporando la voz de los participantes, sus experiencias, actitudes, creencias y reflexiones tal como son esperadas por ellos mismos." Para esta investigación la descripción directa de los hechos y las vivencias de las experiencias con los, estudiantes en cuanto a la enseñanza- aprendizaje de la lengua castellana es el, punto central del proyecto (p.46).

Este enfoque involucra a los docentes y estudiantes durante la investigación a participar activamente en todo el proceso con el propósito de contribuir en la transformación de la realidad, siendo el objetivo principal de este tipo de investigación producir conocimiento y sistematizar las experiencias con el propósito de cambiar una realidad educativa; en este caso específico, el de contribuir al fortalecimiento de las competencias de lenguaje de los estudiantes del grado $8^{\circ}$ en habilidades en lectura crítica para producción de artículos de opinión.

En relación con la población y muestra el proyecto se realizó en la Institución Educativa Vado Castro en el municipio de Tópaga, con 16 estudiantes de grado $8^{\circ}$ entre los 14 y 17 años de edad (7 mujeres 9 hombres), quienes provienen de la parte rural; la actividad económica de los padres está centrada en la agricultura en alto porcentaje, también a la cría de ganado, la minería de carbón y arena y otros hacen parte de las empresas de Holcim y Paz de Rio como empleados del sector privado. De igual manera, presentan grandes necesidades afectivas y económicas; no obstante son personas receptivas y con ganas de salir adelante para ayudar a sus familias, en la mayoría de actividades demostraron buena actitud, fueron atentos y manifestaron actitud positiva frente al cambio.

\section{Resultados}

Para dar respuesta al primer objetivo de la investigación que buscaba identificar el nivel de lectura crítica en la población objeto de estudio, se implementó una prueba diagnóstica (test) con el objetivo de identificar en los estudiantes del grado $8^{\circ}$ el nivel de interpretación y producción textual en
La investigación cualitativa se considera como un proceso activo, sistemático, y riguroso de indagación dirigida en el cual se toman decisiones sobre lo investigable en tanto está en el campo de estudio. 
el que se encuentran. El test consistía en un texto periodístico en el cual los estudiantes debían identificar como primera medida: la tipología del texto, sus características, el tema, interpretar inferencial y literalmente el texto, significados de palabras centrales de la idea del texto periodístico y para finalizar, se proponían varias preguntas donde el lector debía identificar y analizar la intención, punto de vista y argumentaciones del autor. El análisis de los resultados de dicha prueba permitió determinar los siguientes aspectos: escasez de vocabulario, deficiencias en la estructura gramatical, en la interpretación literal e inferencial, campos semánticos y las características del texto y en un $80 \%$ los estudiantes no contestaron las preguntas relacionadas directamente con lectura crítica. También se encontraron dificultades relacionadas con la ortografía en cuanto a puntuación, acentuación $\mathrm{y}$ conectores inadecuados para enlazar las oraciones coherentemente.

En la fase de Aplicación, se diseñó una propuesta pedagógica con base en referentes teóricos y estrategias propias de la lectura crítica, la cual está conformada por 10 talleres, encaminados a promover las competencias lectoras y escritoras, para que el estudiante produzca textos de opinión, que se darán a conocer en un periódico virtual. Una característica esencial fue el trabajo en equipo para lograr la revisión, la reflexión y la realimentación de los diferentes textos que se construyan.

\section{Tabla 1. Plan de acción}

\begin{tabular}{|c|c|c|}
\hline Momentos & Actividades & Tiempo \\
\hline Momento de exploración & -Aplicar taller de Tipología textual & 6 sesiones \\
\hline \multirow{5}{*}{$\begin{array}{l}\text { Se motiva al estudiante a un nuevo } \\
\text { aprendizaje reconociendo sus } \\
\text { saberes previos frente a la temática } \\
\text { a abordar, en este caso los artículos } \\
\text { de opinión. }\end{array}$} & $\begin{array}{l}\text {-Se lee un fragmento de un texto y que ellos } \\
\text { den su punto de vista o reflexión del texto. }\end{array}$ & \\
\hline & $\begin{array}{l}\text {-Después se escoge un párrafo y que ellos } \\
\text { lo escriban con otras palabras la misma } \\
\text { idea. }\end{array}$ & \\
\hline & $\begin{array}{l}\text {-Observar artículos de prensa escrita y } \\
\text { virtual }\end{array}$ & \\
\hline & $\begin{array}{l}\text {-Reconocer partes de los artículos de } \\
\text { opinión }\end{array}$ & \\
\hline & Aplicar taller de gramática & \\
\hline \multirow[t]{3}{*}{ Momento de comprensión } & - Valorar la procedencia del texto & 8 sesiones \\
\hline & - Interrogar al texto & \\
\hline & - Relacionar saberes previos & \\
\hline \multirow{7}{*}{$\begin{array}{l}\text { Se elabora una conceptualización, } \\
\text { enseñanza explicita y modelación } \\
\text { en relación a la lectura crítica y } \\
\text { producción de artículos de opinión. }\end{array}$} & - Contextualizar el texto & \\
\hline & - Diferenciar entre hechos y opiniones & \\
\hline & - Considerar la posición del autor & \\
\hline & - Determinar controversia. & \\
\hline & - Establecer relaciones intertextuales & \\
\hline & - Reconocer analogías & \\
\hline & - Asumir una lectura crítica & \\
\hline
\end{tabular}




\begin{tabular}{|c|c|c|}
\hline \multirow[t]{2}{*}{$\begin{array}{l}\text { Momento de } \\
\text { estructuración }\end{array}$} & $\begin{array}{l}\text {-Seleccionar un artículo de opinión } \\
\text {-Investigar sobre el tema que trata el } \\
\text { artículo }\end{array}$ & 6 sesiones \\
\hline & -Estructurar artículo de opinión a elaborar & \\
\hline \multirow{2}{*}{$\begin{array}{l}\text { Utilizando materiales y } \mathrm{TIC} \text { se } \\
\text { realizan ejercicios donde se aplique } \\
\text { la producción de textos de opinión }\end{array}$} & $\begin{array}{l}\text {-Elaborar la preescritura de un artículo de } \\
\text { opinión }\end{array}$ & \\
\hline & -Hacer correcciones & \\
\hline \multirow[t]{2}{*}{ Momento de realimentación } & $\begin{array}{l}\text {-Realizar lectura y debates de los textos } \\
\text { escritos por los estudiantes para ayudar en } \\
\text { su construcción. }\end{array}$ & 5 sesiones \\
\hline & -Elaborar la exposición de trabajos & \\
\hline \multirow[t]{2}{*}{ Momento de valoración } & $\begin{array}{l}\text {-Exponer en el periódico mural los textos } \\
\text { de opinión }\end{array}$ & 4 sesiones \\
\hline & $\begin{array}{l}\text {-elaborar un periódico con los textos } \\
\text { de opinión de los chicos utilizando } \\
\text { PUBLISHER }\end{array}$ & \\
\hline
\end{tabular}

Fuente. Autoría propia, excepción el momento de comprensión que es basado en Girón, Jiménez \& Camargo (2007, p.50).

Respecto a la producción textual, se tuvo especial cuidado con aspectos fundamentales en cuento a cohesión y coherencia, en las cuales el estudiante reconoció la estructura de cada uno de los artículos de opinión. De igual forma, fue importante que utilizaran adecuadamente la sintaxis del lenguaje, la cohesión y la coherencia y sea completamente autónomo de sus opiniones y reflexiones a que llega después de haber leído determinados textos, realizado consultas exhaustivas acerca de temas relevantes que impliquen una profunda indagación que pueda aportar información verídica que beneficie a la sociedad en un conocimiento significativo y relevante para enriquecer las posibles incertidumbres de los sujetos receptores de información que tienen a su alrededor.

Luego de la implementación de la propuesta se concentraron los esfuerzos en analizar los avances que se hicieron respecto a tres aspectos: compresión lectora, lectura crítica y producción escrita; Al ser la lectura parte fundamental del proyecto, el primer gran logro fue acercar a los jóvenes de grado octavo a la lectura de textos informativos que les permitiera conocer su realidad, analizarla y tomar una postura crítica frente a la misma, al tiempo que esto permitió al docente confirmar que la lectura permite aprehender y comprender, "leer es construir una interpretación y una comprensión personal de dicho texto" (Solé, 1996). Es importante tener objetivos para la lectura, argumenta Solé (1996):

Si el lector sabe que significa leer, y se pregunta al hacer una lectura, qué lee, por qué lee y para qué lee, va a estar motivado para entender el significado de dicha lectura y para hacerlo se ayudará de su propio bagaje de conocimientos, 
La producción escrita se motivó a través de la reflexión y la autocrítica, durante el análisis de resultados se tuvo en cuenta las etapas de los talleres que permitieron al estudiante hacer pequeñas producciones; al mismo tiempo, el docente observó paso a paso la forma en que los estudiantes avanzaron en los diferentes aspectos de la producción. es decir de lo que ya conoce y sabe que son sus conocimientos previos (p.67).

En consecuencia, el acercamiento a la realidad a través de los textos les permitió a los jóvenes reflexionar y esto se vio reflejado en los artículos de opinión escritos por ellos y en las diferentes discusiones que se tuvieron durante el desarrollo de las sesiones de clase en las cuales se dialogó en torno a los artículos y los temas tratados por diferentes autores consultados por ellos o por el docente.

En cuanto al análisis textual se notó que a medida que los estudiantes se familiarizan con los artículos de opinión y los textos informativos comenzaron a hacer un avance notorio en cuanto a la inferencia, la organización lógica y la secuenciación de textos; en relación con la lectura crítica los estudiantes asumieron una actitud mucho más reflexiva frente a ella.

Además de lo anterior, se hizo el análisis de resultados de los talleres aplicados, los cuales determinaron en gran medida el avance de la investigación. Desde su propio diseño los talleres permitieron que los estudiantes asumieran una actitud responsable y autónoma frente a la lectura y la producción escrita. En la aplicación y el análisis de los talleres evidenció que los estudiantes gradualmente adquirieron habilidad para organizar ideas, escribir secuencias, utilizar diferentes recursos lingüísticos necesarios y así mismo se les motiva a terminar tareas en tiempos determinados, al respecto Rodríguez
(2012) indica que: “...su puesta en marcha promueve el diálogo entre los participantes, la exposición libre de los puntos de vista para la negociación de las acciones a seguir, la definición de los propósitos comunes, las funciones de los miembros, las metas por alcanzar y los medios requeridos para lograrlo; fomenta también el despliegue de estrategias discursivas como el diálogo, la narración, la explicación y la argumentación, entre otras, de acuerdo con los propósitos y acciones comunicativas que orientan el sentido del taller”(p.7).

La producción escrita se motivó a través de la reflexión y la autocrítica, durante el análisis de resultados se tuvo en cuenta las etapas de los talleres que permitieron al estudiante hacer pequeñas producciones; al mismo tiempo, el docente observó paso a paso la forma en que los estudiantes avanzaron en los diferentes aspectos de la producción. El resultado más evidente fue el artículo que presentó cada estudiante al final del proceso, que a pesar de presentarse falencias propias de la escritura escolar como faltas ortográficas y de puntuación, se puede afirmar que los estudiantes realmente comprendieron la importancia de los artículos de opinión como excusa para comprender su propia realidad a través de la reflexión. La producción se vio reflejada en la publicación de los artículos en un periódico mural en la institución, por medio de este se logró que cada estudiante pudiera socializar su producción a la comunidad educativa y al mismo tiempo de la producción escrita 
hizo que se reforzara la competencia argumentativa. Esta actividad se convirtió en una oportunidad de validar del conocimiento producido, es importante que los estudiantes comprendan que al producir conocimiento debe ser compartido en la comunidad para que este sea válido, sin validación no existe avance intelectual. El análisis de resultados se hizo de manera más reflexiva que de manera cuantitativa ya que, el fin último de la investigación estaba relacionado con la capacidad argumentativa de los estudiantes, para hacerlo se tuvieron en cuenta los procesos evaluativos propios y los avances visto personalmente por el docente investigador.

\section{Conclusiones}

Se evidenció durante el diagnóstico frente a la evaluación de la lectura y la escritura, que los estudiantes presentan deficiencias en sus escritos puesto que no son claros ni explícitos, es decir, a pesar de que se dan instrucciones generales, el estudiante presenta interrogantes y bajos conocimientos en el léxico propio del área y poco vocabulario. En este caso las estrategias didácticas deberían orientarse a considerar la escritura como un proceso que requiere distintas etapas: planificación, redacción, revisión y rescritura, con distintos lectores, para la construcción de saberes, el pensamiento crítico y no como una práctica de control.

Al tener en cuenta el PEI de la Institución se observa la necesidad de consolidar estrategias en el aula para consolidar los procesos de escritura y lectura crítica, pues si bien existen lineamientos curriculares de la transversalidad de las competencias comunicativas, en lo pedagógico se requiere abrir espacios de discusión y formación.

La propuesta pedagógica desarrollada brindó las estrategias didácticas para el desarrollo de habilidades en la lectura crítica para escribir textos de opinión ya que es importante incrementar la escritura de artículos de opinión, promoviendo la lectura crítica de una manera transversal; para ello se requiere de un fortalecimiento en los docentes sobre la importancia que tiene la lectura y la escritura en los estudiantes para su formación académica.

Es importante realizar investigaciones que ayuden a mejorar la calidad de la educación en Colombia y a cumplir con estándares del conocimiento para tener profesionales competentes. Formar estudiantes con una actitud crítica a la sociedad es un reto de la educación, la lectura crítica debe ser un objetivo prioritario del currículo, la habilidad de ser un lector crítico es inherente a las personas y a las sociedades.

La lectura crítica y el pensamiento crítico son construcciones culturales que necesitan educación, esfuerzo y cultivo permanente. Se debe brindar al estudiante diversas estrategias de lectura crítica que le permitan descubrir el punto de vista que los discursos reflejan de la realidad, porque lo que aprendieron en la educación previa les resulta insuficiente cuando se enfrentan al aprendizaje de las disciplinas. Leer críticamente desarrolla habilidades 
que ayudan a la adquisición del conocimiento, ayudándole en su autonomía, criterio propio y dominio para argumentar y ayudar en el proceso educativo.

Las prácticas pedagógicas innovadoras ayudan a que los estudiantes se emocionen por el aprendizaje del lenguaje, al crear estrategias para potencializar diferentes habilidades del pensamiento y fomentar los niveles de calidad en el área de lenguaje, la lectura crítica es una estrategia importante para desarrollar el gusto por la lectura, al desarrollar la competencia comunicativa y ayuda a construir realidades ejerciendo autonomía, valores democráticos y sociales que se esperan de los ciudadanos de hoy.

La escuela debe propiciar espacios que faciliten la adquisición de hábitos de lectura y la concentración en cuanto tiempo y duración, permitiendo a los estudiantes ser partícipes de la creación mental apoyando su esfuerzo en el constante trabajo por entender la realidad y dar soluciones a la problemática del contexto.

\section{Referencias}

Cassany, D (1993). La Cocina de la escritura. Barcelona: Editorial Anagrama.

Díaz-Barriga \& Hernández (2012). Estrategias Docentes para un aprendizaje significativo, una interpretación constructivista. México: Editorial Mc Graw Hill.

Gialdino, I (2006). Estrategias de Investigación cualitativa. Barcelona, España, p. 163.

Girón,S., Jimenez, C. \& Lizcano ,C. (2007). Colección Cuadernillo serie gramática. Universidad Sergio Arboleda, p.45-123.

Jurado, F (2014). La lectura crítica. El dialogo entre los textos. Revista Santillana, (8), 12.

Kemmis, S (1998). El Currículo más allá de la teoría de la reproducción. Madrid: Morata.

Solé, I (1996). Estrategias de lectura. Barcelona: Editorial Graó.

Solé, I (1998). Estrategias de Lectura. Barcelona: Editorial Graó.

Tobón, L. (2007). La lingüistica del lenguaje. Bogotá: Fondo Editorial de la Universidad Pedagógica Nacional. 